\title{
CBDCA + Pemetrexed + Bevacizumab and Its Maintenance Chemotherapy in a Case of Solitary Breast Metastasis from a Lung Adenocarcinoma Resistant to Gefitinib
}

\author{
Kazuhide Sato ${ }^{a}$ Yoshihiro Takeyama $^{a}$ Motoi Yoshihara $^{b}$ \\ Toshio Kato $^{\text {a Hiroyuki Hashimoto }}{ }^{a}$ Yasutaka Fukui $^{\mathrm{a}}$ Hideo Gonda ${ }^{a}$ \\ Ryujiro Suzuki ${ }^{a}$ \\ Departments of ${ }^{a}$ Respiratory Medicine and ${ }^{b}$ Surgery, Toyohashi Municipal Hospital, Toyohashi, \\ Japan
}

\section{Key Words}

Solitary breast metastasis - Non-small-cell lung carcinoma - T790M .

CBDCA + pemetrexed + bevacizumab - Maintenance therapy .

Tyrosine kinase inhibitor resistance

\begin{abstract}
Based on the AVAPERL trial (36th ESMO 2011), CBDCA + pemetrexed + bevacizumab and its maintenance chemotherapy with pemetrexed + bevacizumab is a new promising regimen for the treatment of advanced non-small-cell lung adenocarcinoma. Herein, we report the rare case of a patient with solitary breast metastasis from a lung adenocarcinoma, which was effectively treated using CBDCA + pemetrexed + bevacizumab and its maintenance chemotherapy. A 57-year-old female was admitted to the hospital due to pleural effusion and cardiac tamponade caused by a lung adenocarcinoma possessing a mutation of the epidermal growth factor receptor (EGFR) gene (deletion of exon 19). The patient was treated by first-line chemotherapy (gefitinib $250 \mathrm{mg} /$ body/day) which resulted in complete response. After 12 months, carcinoembryonic antigen was gradually increasing and she complained of a right breast mass. With a core-needle biopsy, the breast tumor was pathologically diagnosed as recurrence and solitary metastasis of a lung adenocarcinoma. Further study of the second mutation of EGFR revealed a T790M mutation. The patient was treated by second-line chemotherapy [CBDCA + pemetrexed + bevacizumab (AUC $6+500$ $\mathrm{mg} / \mathrm{m}^{2}+15 \mathrm{mg} / \mathrm{kg}$ )] and its maintenance chemotherapy (pemetrexed + bevacizumab). The cases of patients with breast metastasis from other organs are very rare.
\end{abstract}


Immunohistopathological analysis is very useful to diagnose whether the malignancy is primary or not. In the case of a breast tumor with present or previous malignancy, a metastatic breast tumor should be considered. Furthermore, the biopsy of the breast metastasis also revealed the second mutation of resistance to gefitinib, T790M. Of note, according to our case, CBDCA + pemetrexed + bevacizumab and its maintenance chemotherapy is feasible and well tolerated for breast metastasis from a lung adenocarcinoma which is resistant to gefitinib and possesses the T790M mutation in the EGFR gene.

\section{Introduction}

Metastasis to an unusual site is much less frequent with non-small-cell lung carcinoma (NSCLC) and the incidence of metastasis to the breast is even lower [1-3]. Solitary metastasis to the breast from a lung carcinoma has never been reported before in Japan and only a few cases have been reported worldwide [2]. The precise diagnosis of breast metastasis from the lung is difficult due to its rarity. Herein, we present a case of solitary breast metastasis from a lung adenocarcinoma. The patient had been in the situation of complete response of NSCLC, therefore it was an important issue whether the mass of breast metastasis was primary or not. Pathological analysis with immunostaining was useful for a precise diagnosis [4]. Furthermore, the biopsy of the breast metastasis revealed the second mutation of resistance to gefitinib in the epidermal growth factor receptor (EGFR) gene (T790M). Satisfying improvement of the breast metastasis by the treatment with CBDCA + pemetrexed + bevacizumab and its following maintenance therapy was also observed.

\section{Case Presentation}

A 57-year-old female Japanese patient was admitted to our hospital because of dyspnea. Her previous medical history was unremarkable; she was a housewife with no history of smoking or alcohol use. Positive physical examination revealed a blood pressure of 160/100 $\mathrm{mm} \mathrm{Hg}$, a heart rate of 100 beats/min, SpO2 92\% (2 liter nasal cannula), right neck lymph node swelling and bibasilar crackle. Chest X-ray showed bilateral pleural effusions, consolidations in the right upper lung and dilated cardio thoracic ratio (fig. 1 a). Chest CT of the upper lesions showed pleural effusions and swelled lymph nodes in mediastinum (fig. 1b). Chest CT of the lower lesions showed cardiac effusions which caused cardiac tamponade (fig. 1c). Abdominal CT showed multiple metastases in the liver. Cytopathological analysis of the pleural and cardiac effusions revealed that the patient suffered from a lung adenocarcinoma possessing a mutation of the EGFR gene (deletion of exon 19, fig. 1d). The clinical stage of the NSCLC was T2N3M1b (stage IV). Drainage of the pleural and cardiac effusions was performed and the patient was treated by first-line chemotherapy (Gefitinib $250 \mathrm{mg} /$ body/day). No severe adverse events were observed. After 7 months, chest CT showed no pleural and cardiac effusion (fig. 1e). The liver metastases lesions had disappeared. Chest CT of the upper lesions showed that swelled lymph nodes in mediastinum had also disappeared (fig. 1f). The response was determined as complete response, but carcinoembryonic antigen (CEA) was gradually increasing and the patient complained of a right breast mass 12 months after the introduction of Gefitinib.

Mammography showed calcifications in both mammae (fig. 1g). Chest CT of the breast lesions showed that the right swelled mammary mass was contrasted (fig. 1h). Chest CT showed no pleural and cardiac effusions, and the liver metastases lesions were not recurrent. Whole-body bone scintigraphy showed a high intensity in the right breast lesion (fig. 1i). Mammary ultrasonography showed an occupying lesion in the right breast and multiple swelled right axillary lymph nodes (fig. $1 \mathrm{j}, \mathrm{k}$ ). The left breast and axillary lymph nodes showed no abnormalities. Pathological analysis of the needle biopsy 
of the mass revealed it as an adenocarcinoma (fig. 2a). To clarify whether the breast mass was primary or not, additional immunohistochemical analysis was performed. Immunohistochemical analysis showed cytokeratin 7-positive (CK7+), CK20-, thyroid transcription factor 1-positive (TTF$1+$ ), surfactant protein A-positive (SP-A+), Mucin 1-positive (MUC1+), MUC2-, gross cystic disease fluid protein-15-negative (GCDFP15-), estrogen receptor-negative (ER-), progesterone receptornegative (PgR-) and human epidermal growth factor receptor type 2-negative (HER2-; fig. 2b-k), indicating that the primary origin of the mass was not the breast but the lung. Furthermore, we found the mutation of resistance to gefitinib, T790M, thanks to the biopsy of the breast metastasis. Taken together these results, the mass was due to recurrence and metastasis from the lung. Because the type of the mass was classified as an inflammatory breast tumor, we assumed that a surgical approach was difficult. With these considerations, we determined to treat the patient by second-line chemotherapy [CBDCA + pemetrexed + bevacizumab (AUC $6+500 \mathrm{mg} / \mathrm{m}^{2}+15 \mathrm{mg} / \mathrm{kg}$ )] and its following maintenance chemotherapy (pemetrexed + bevacizumab). After 4 courses of CBDCA + pemetrexed + bevacizumab, mammary ultrasonography showed a decrease in the size of the tumor in the right breast and decreased multiple swelled right axillary lymph nodes (fig. $3 \mathrm{a}, \mathrm{b}$ ). Chest CT of the breast lesions showed that the right swelled mammary tumor was also decreasing (fig. 3c). The patient mentioned that the mass had disappeared. The effectiveness was determined as partial response. The patient underwent maintenance chemotherapy was undergone for 8 months after the induction of second-line chemotherapy and no evidence of worsening has been seen until today. The overview of the therapy and change of CEA is shown in fig. 4.

\section{Discussion}

Worldwide, lung cancer is the most common malignancy and the leading cause of cancer death (1.6 million new cases per year and 1.378 million deaths, accroding to the International Agency for Research on Cancer). Adenocarcinoma accounts for $40 \%$ of all NSCLCs, and approximately $20 \%$ of all newly diagnosed lung adenocarcinomas present with distant metastases [5]. The most common sites of metastasis in decreasing order are: brain, bone, liver, and adrenal glands [5].

Initially, the breast has some resistance to metastasis from extramammary malignancy because it contains large areas of fibrosis tissue with a relatively poor blood supply. For this reason, breast metastases from extramammary malignancies are rare, accounting for only $0.4-1.3 \%$ of all breast malignancies $[1,2]$.

In the previous international literature, it has been reported that the most common sources of primary tumor metastasis to the breast in decreasing order are: hematological malignancies, malignant melanoma, lung carcinomas, gynecological malignancies, and gastrointestinal malignancies $[1,2,6]$.

A review of the literature (1990-2011) showed approximately 30 NSCLCs as case reports or part of a series of secondary breast tumors [5]. Almost all cases were multiple organ metastases, and only 4 cases were solitary metastases to the breast [2]. Thirteen of the above cases were classified as adenocarcinomas. Other histological types of NSCLCs have also been reported. Radiologic findings of metastatic lesions from extramammary neoplasms showed that $70 \%$ of the cases were single lesions and about $20 \%$ were bilateral with multiple lesions [7]. The most frequent site of metastasis was reported to be the upper outer quadrant [7]. Our case was definitely suitable to these data. Unlike in primary breast tumors, in the majority of metastases there was no retraction of the skin or nipples [7].

Metastasis to the breast is considered to occur by two distinct routes: lymphatic and hematogenous. The features of the metastasis differ in each type. Regarding the 
radiographic appearance of hematogenous metastasis from extramammary malignancies, a solitary discrete lesion is more commonly reported than multiple or diffuse involvement. Lymphatic metastasis to the breast usually occurs across the anterior chest wall to the opposite breast. In our case, the appearance of the lymphatic metastasis type was indistinguishable from that of an inflammatory carcinoma. Our case showed both solitary and inflammatory appearances which are possessing features of both routes.

Because the treatment differs, it is essential to distinguish whether the breast mass is primary or not [4]. However, it is widely known that findings of mammography and ultrasonography are not useful to distinguish this [7]. The most useful tool is the histological and the following immunohistochemical diagnosis. When performing an immunohistochemical analysis, it is important to remember that no marker is $100 \%$ specific or sensitive. Thus, a combination of the immunostainings is recommended. Both immunostainings of CK7 and CK20 are useful to categorize carcinomas; however, differential cytokeratin staining generally does not distinguish breast from lung carcinomas as most adenocarcinomas from both sites are CK7-/CK20- [4]. TTF-1 has been considered to be a useful marker to identify a tumor of thyroid and lung origin and it is expressed in about $80 \%$ of all lung adenocarcinomas. SP-A is also specific to the lung and expressed in approximately $45 \%$ of all lung adenocarcinomas. Other markers considered to be supportive in determining a breast origin are: ER (expressed in $80 \%$ of all breast carcinomas), GCDFP-15 (in 50\%), PgR (in 60\%) and HER2 (in $30 \%$ ). TTF-1 expression in a tumor has traditionally been thought of as excluding the possibility of a breast origin [8]. However, there is one paper describing that TTF-1 expression is seen in a small proportion of breast carcinomas $(2.4 \%, 13 / 546$ cases) [8]. In our case, in combination with other markers, it was possible to make an relatively precise diagnosis.

In patients with NSCLC whose tumors possess an EGFR mutation, first-line tyrosine kinase inhibitor (TKI) therapy is recommended [9]. Multiple studies have shown that patients with NSCLC possessing an EGFR mutation have an approximately 70\% RECIST (Response Evaluation Criteria in Solid Tumors) response rate to EGFR TKIs. However, patients with NSCLC possessing an EGFR mutation develop disease progression after a median of 10 to 14 months on TKI [10]. Unfortunately, no optimal therapy thereafter has yet been established. In our case, a biopsy ('re-biopsy') revealed the T790M mutation in the EGFR gene, which was widely known as a second mutation resistant to EGFR TKIs, such as gefitinib and erlotinib. The current standard practice is to switch to conventional cytotoxic chemotherapy. Because we discontinued TKI to switch to conventional cytotoxic chemotherapy, there was the possibility of accelerated progression (so-called 'flare') [11]. Fortunately, in our case, the switch from TKI to conventional cytotoxic chemotherapy happened smoothly and without 'flare'.

Nowadays, it is essential to make a re-biopsy after NSCLC has become resistant to TKIs, because it was reported that whether the secondary mutation is T790M or not is very important [10]. Several groups studying the molecular biology of acquired resistance have found that at least $50 \%$ the of cases are attributable to a secondary mutation (T790M) in exon 20 of EGFR. The presence of T790M defines a clinical subset with a relatively favorable prognosis and more indolent progression, making the T790M mutation one of the molecular biomarkers [12]. Therefore, the results of investigations regarding whether T790M affects survival have been highly anticipated. 
Overall, metastasis to the breast has been associated with poor prognosis with most patients dying within 1 year after diagnosis [7]. It was reported that the median duration of survival from diagnosis of breast metastasis was 10 months (range 0.4192.7). Patients who had surgery had a median survival duration of 15.5 months, whereas their cohorts who did not have surgery survived only for a median of 8.1 months [6]. In our case, a favorable prognosis was expected because of solitary metastasis and T790M secondary mutation [10].

We tried to treat our patient with second-line chemotherapy [CBDCA + pemetrexed + bevacizumab (AUC $6+500 \mathrm{mg} / \mathrm{m}^{2+} 15 \mathrm{mg} / \mathrm{kg}$ )] and its maintenance chemotherapy (pemetrexed + bevacizumab). Surgical treatment was also a possible therapy, but we decided to perform the chemotherapy after discussions with surgeons specialized in breast carcinomas, because the type of the breast tumor was inflammatory and there were also axillary lymph node swellings. The CBDCA+ pemetrexed +bevacizumab regimen is based on the AVAPERL trial and expected to be a new promising regimen (36th ESMO 2011) [13, 14]. A single-institution experience report showed that the regimen and its maintenance therapy is effective for advanced NSCLC, with a response rate of $52 \%$, acceptable toxicity and promising early survival [15]. With this regimen, our patient achieved good PR and the breast mass disappeared.

\section{Conclusions}

We reported a rare case of a patient with solitary breast metastasis from a lung adenocarcinoma which was effectively treated using CBDCA + pemetrexed + bevacizumab and its maintainance chemotherapy. Of note, according to our case, CBDCA + pemetrexed + bevacizumab and its maintenance chemotherapy is feasible and well tolerated for solitary breast metastasis from a lung adenocarcinoma which is resistant to gefitinib and possesses the secondary T790M mutation in the EGFR gene.

\section{Acknowledgement}

We are grateful to Dr. Matuyoshi Maeda for his clinical pathological knowledge, and Dr. Shigehisa Kajikawa at Nagoya University Graduate School of Medicine for his help with the first therapy. We also thank our previous co-workers, Dr. Yogo and Dr. Ohdate. This work was supported by the Central Japan Lung Study Group.

\section{Disclosure Statement}

None of the authors have a conflict of interest to declare. 

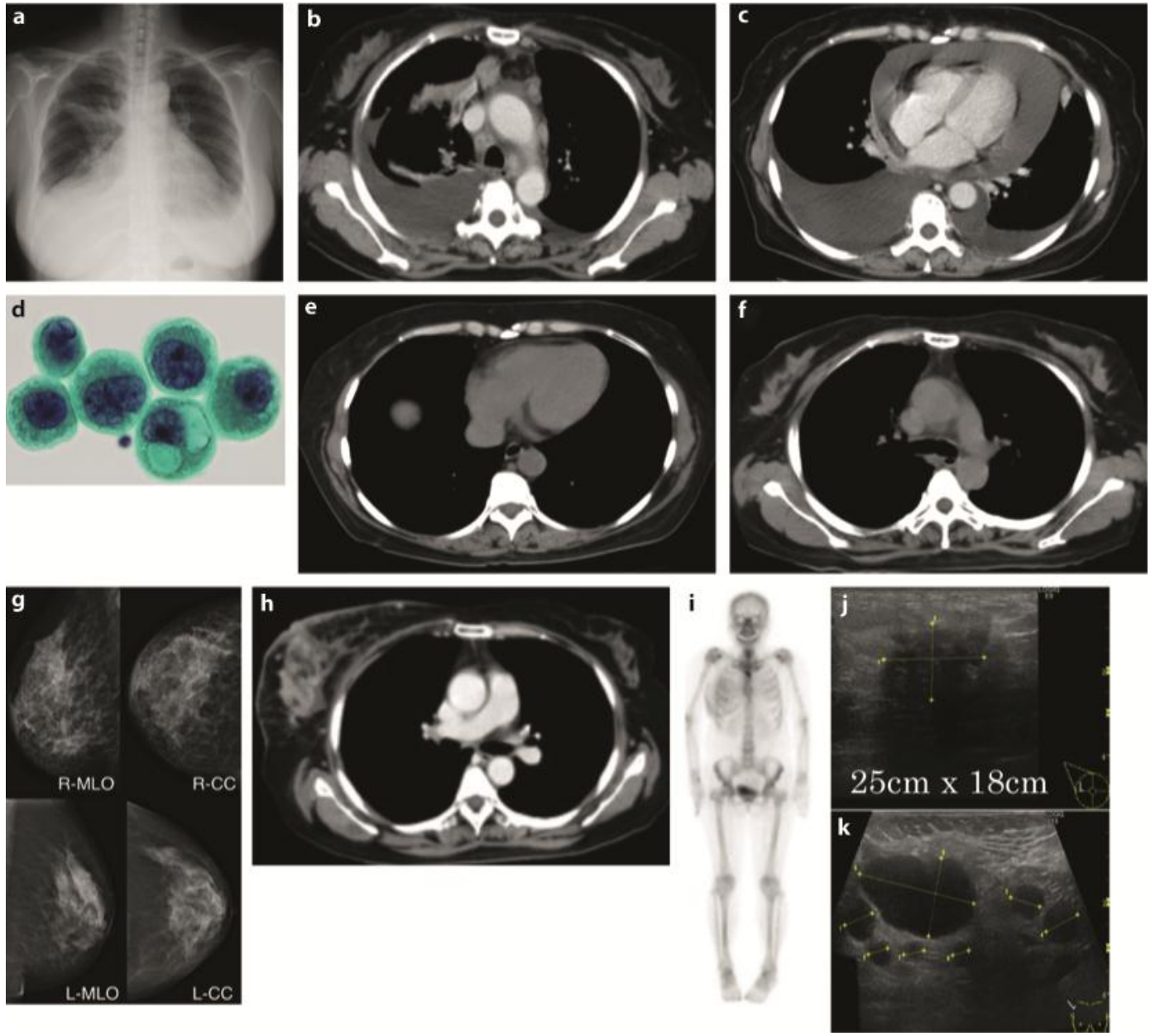

Fig. 1. X-ray, CT and cytopathological findings. a X-ray on admission. b, c CT on admission. d Papanicolaou stain of pleural effusion. e, $\mathbf{f}$ CT after first-line chemotherapy. g Mammography at recurrence. $\mathbf{h}$ Chest CT of the breast at recurrence. i Scintigraphy at recurrence. $\mathbf{j}$ Mammary ultrasonography of the right breast at recurrence. $\mathbf{k}$ Ultrasonography of the right axillary at recurrence.
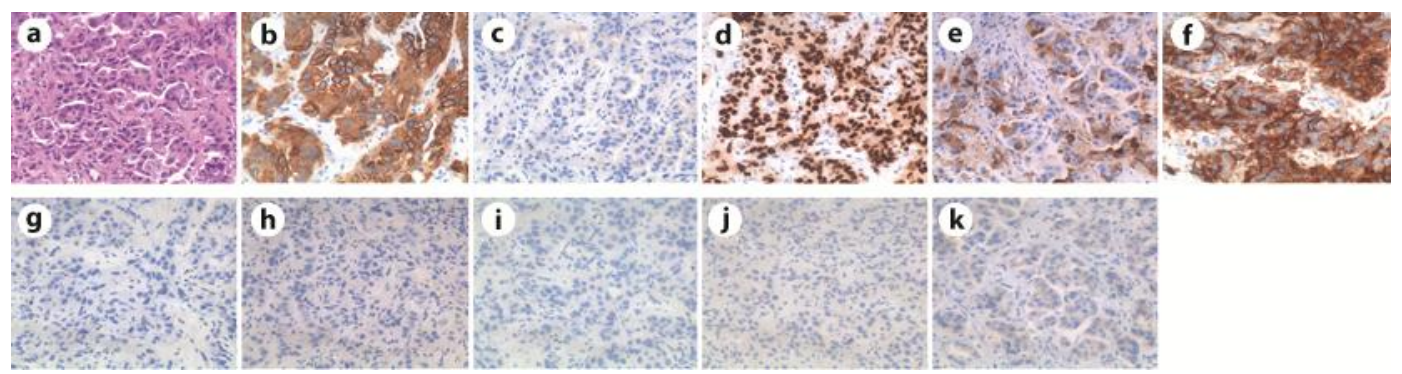

Fig. 2. Pathological analysis of the needle biopsy. a Hematoxylin-eosin staining. Immunostaining of CK7 (b). CK20 (c), TTF-1 (d), SP-A (e), MUC1 (f), MUC2 (g), GCDFP15 (h), ER (i), PgR (j) and HER2 (k). 

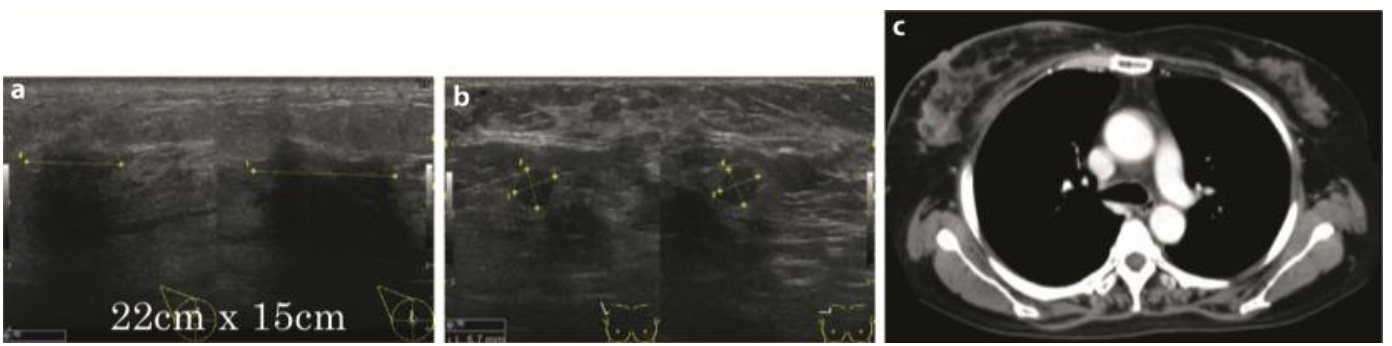

Fig. 3. a Mammary ultrasonography of the right breast after second-line therapy. b Ultrasonography of the right axillary after second-line therapy. c Chest CT of the breast after second-line therapy.

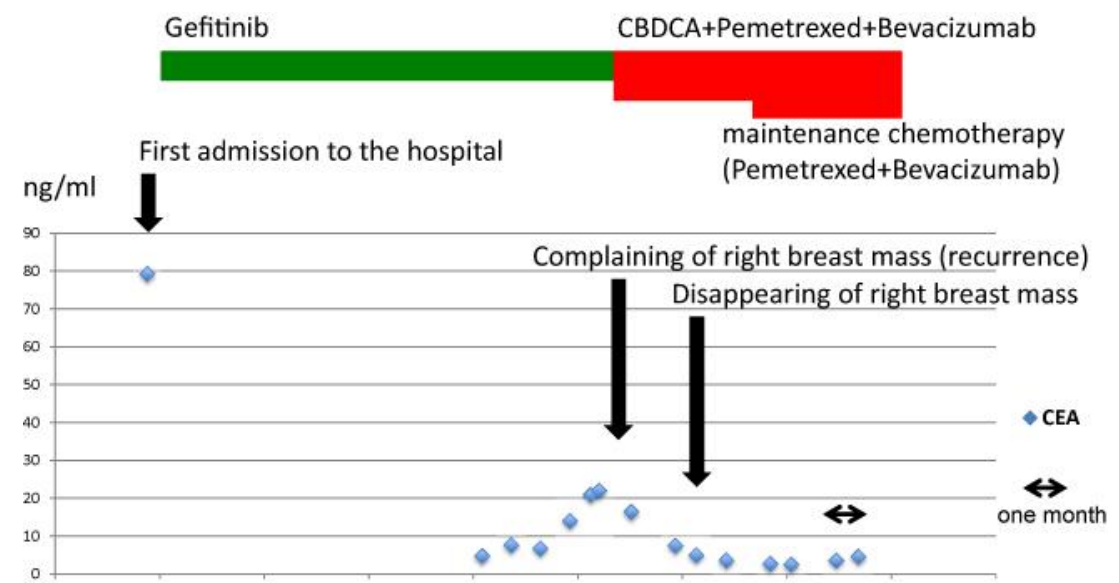

Fig. 4. Overview of the therapy and change of CEA.

\section{References}

1 Georgiannos SN, Chin J, Goode AW, Sheaff M: Secondary neoplasms of the breast: a survey of the 20th century. Cancer 2001;92:2259-2266.

$\checkmark 2$ Lee SK, Kim WW, Kim SH, et al: Characteristics of metastasis in the breast from extramammary malignancies. J Surg Oncol 2010;101:137-140.

-3 Ramar K, Pervez H, Potti A, Mehdi S: Breast metastasis from non-small-cell lung carcinoma. Med Oncol 2003;20:181-184.

4 Lee AH: The histological diagnosis of metastases to the breast from extramammary malignancies. J Clin Pathol 2007;60:1333-1341.

5 Maounis N, Chorti M, Legaki S, et al: Metastasis to the breast from an adenocarcinoma of the lung with extensive micropapillary component: a case report and review of the literature. Diagn Pathol 2010;5:82.

6 Williams SA, Ehlers RA, Hunt KK, et al: Metastases to the breast from nonbreast solid neoplasms: presentation and determinants of survival. Cancer 2007;110:731-737.

7 Yeh CN, Lin CH, Chen MF: Clinical and ultrasonographic characteristics of breast metastases from extramammary malignancies. Am Surg 2004;70:287-290.

-8 Robens J, Goldstein L, Gown AM, Schnitt SJ: Thyroid transcription factor-1 expression in breast carcinomas. Am J Surg Pathol 2010;34:1881-1885.

9 Maemondo M, Inoue A, Kobayashi K, et al: Gefitinib or chemotherapy for non-small-cell lung cancer with mutated EGFR. New Engl J Med 2010;362:2380-2388. 
10 Oxnard GR, Arcila ME, Sima CS, et al: Acquired resistance to EGFR tyrosine kinase inhibitors in EGFRmutant lung cancer: distinct natural history of patients with tumors harboring the T790M mutation. Clin Cancer Res 2011;17:1616-1622.

11 Chaft JE, Oxnard GR, Sima CS, et al: Disease flare after tyrosine kinase inhibitor discontinuation in patients with EGFR-mutant lung cancer and acquired resistance to erlotinib or gefitinib: implications for clinical trial design. Clin Cancer Res 2011;17:6298-6303.

12 Oxnard GR, Arcila ME, Chmielecki J, et al: New strategies in overcoming acquired resistance to epidermal growth factor receptor tyrosine kinase inhibitors in lung cancer. Clin Cancer Res 2011;17:5530-5537.

13 Stevenson JP, Langer CJ, Somer RA, et al: Phase 2 trial of maintenance bevacizumab alone after bevacizumab plus pemetrexed and carboplatin in advanced, nonsquamous nonsmall cell lung cancer. Cancer 2012, E-pub ahead of print.

14 Patel JD, Bonomi P, Socinski MA, et al: Treatment rationale and study design for the pointbreak study: a randomized, open-label phase III study of pemetrexed/carboplatin/bevacizumab followed by maintenance pemetrexed/bevacizumab versus paclitaxel/carboplatin/bevacizumab followed by maintenance bevacizumab in patients with stage IIIB or IV nonsquamous non-small-cell lung cancer. Clin Lung Cancer 2009;10:252-256.

15 Malhotra B, Evans T, Weiss J, et al: Carboplatin/pemetrexed/bevacizumab in the treatment of patients with advanced non-small-cell lung cancer: a single-institution experience. Clin Lung Cancer 2010;11:192-197. 